\title{
Influence of Dosimetric Considerations in Evaluating Second Cancer Risks in Prostate Cancer
}

\author{
Raghda A. Elgendy' ${ }^{1}$ Wahib M. Attia², Ehab M. Attalla3 ${ }^{3}$, Mostafa A. Elnaggar ${ }^{1,4}$ \\ ${ }^{1}$ Medical Physics Department, Ayady Al Mostakbal Oncology Center, Alexandria, Egypt \\ ${ }^{2}$ Physics Department, Faculty of Science, Suez Canal University, Ismailia, Egypt \\ ${ }^{3}$ Radiotherapy and Nuclear Medicine Department, National Cancer Institute, Cairo University, Giza, Egypt \\ ${ }^{4}$ Cancer Management and Research Department, Medical Research Institute, Alexandria University, Alexandria, Egypt \\ Email: raghdaelgendy_197@yahoo.com
}

How to cite this paper: Elgendy, R.A., Attia, W.M., Attalla, E.M. and Elnaggar, M.A. (2018) Influence of Dosimetric Considerations in Evaluating Second Cancer Risks in Prostate Cancer. International Journal of Medical Physics, Clinical Engineering and Radiation Oncology, 7, 203-213.

https://doi.org/10.4236/ijmpcero.2018.72018

Received: April 18, 2018

Accepted: May 25, 2018

Published: May 28, 2018

Copyright $\odot 2018$ by authors and Scientific Research Publishing Inc. This work is licensed under the Creative Commons Attribution International License (CC BY 4.0).

http://creativecommons.org/licenses/by/4.0/

\begin{abstract}
Influence of dosimetric considerations in evaluating second cancer risks in prostate cancer. Material and methods: Fifteen patients in this study suffering from early stage of prostate cancer, each patient underwent three plans: 1) Three-dimensional conformal radiation therapy (3DCRT), 2) Rotation therapy (Arc therapy), and 3) intensity-modulated radiation therapy (IMRT) plan. Estimate secondary metastasis risk models: Excess Relative Risk (ERR) and Excess absolute risk (EAR) based on age of exposure by taking dosimetry data from Dose Volume Histograms (DVHs) to calculate risk models. Result: The second cancer risk models (ERR and EAR) for organs at risk OARs decrease with increasing age of exposure for $3 \mathrm{D}-\mathrm{CRT}, \mathrm{ARC}$ and IMRT and there is no significant difference for ERR and EAR model for developing second cancer risk in 3D-CRT, ARC and IMRT.
\end{abstract}

\section{Keywords}

Prostate Cancer, Second Cancer Risks, 3DCRT, Rotational Arc and IMRT

\section{Introduction}

Radiotherapy aims to give the prescribed dose to the tumor and to protect as much as possible to organs at risk and surrounded healthy tissue. The radiotherapy nowadays, together with chemotherapy and surgery, is a way to treat the patients who have different kind of tumors [1].

Prostate cancer is the leading cancer in men after the age of 50, which is a real public health problem. According to the data of 2005 cancer registry of the Rabat 
region (RECRAB), prostate cancer is the second most common cancer in men after lung cancers with standardized incidence to the world population. Ten-year's US data from the SEER program show relative survival of 91.7\% [2].

Prostate cancer has reported either an increased risk of secondary malignancies or no relation to estimate the secondary cancers risk after radiotherapy and negligible risk of secondary malignancies after radiotherapy from other previous review where it is important for both patients and physicians to consider the risk [3].

Radiotherapy has an important role in the treatment of prostate cancer. Three-dimensional conformal radiation therapy (3D-CRT), intensity modulated radiation therapy (IMRT) and volumetric modulated arc therapy (VMAT) techniques are all applied for this purpose [4].

The importance of dose escalation for tumor control in the management of localized prostate cancer after External beam radiotherapy (EBRT) as a definitive treatment has been shown in numerous trials where by increasing the radiation dose then the developing complications caused by injury to organs at risk (bladder, prostatic urethra and rectum) also increase [5].

Rotation Therapy technique or ARC therapy is the treatment delivery whereas gantry rotates around the patient, it looks like an infinite extension of the multiple-field technique. The most useful of this technique is when applied to small, symmetric, deep-seated tumors and limited to the treatment of centrally located lesions (i.e., an equal amount of tissue in all directions around the lesion). To reduce dose to critical normal structures one or more sectors of a 360-degree rotation are skipped then the high-dose region is shifted away from the skipped region. ARC therapy dose distributions are not very sensitive to the energy of the photon beam [6].

Intensity-modulated radiotherapy (IMRT) has shown to significantly reduce acute toxicity rates compared with what has been observed with 3D-CRT [7].

Intensity modulated radiation therapy allows conformity to the tumor while saving the adjacent normal structures. IMRT generates high gradient doses on the target, with rapid drop in the latter to the level of normal structures, result of IMRT technique requires extremely high precision in treatment but with very minor positioning errors can affect the target cover and increase the dose to organs at risk (OARs] [8].

Is It expect that 220,800 new cases of prostate cancer and around 27,540 deaths from estimation at 2015. Prostate cancer is the second most common cancer and the second leading cause of cancer death for men in the United States. External beam radiation therapy is commonly used to treat prostate cancer. Studies have shown the benefits of 76 Gy or higher conventionally fractionated treatments, although there is a substantial risk of gastrointestinal toxicity, particularly stemming from the rectum dose. In these cases, radiation doses better conforming to the prostate are necessary to reduce possible rectal complications [9].

\section{Material and Methods}

Fifteen patient in this study suffering from early stage of prostate cancer, Selec- 
tion process based on low staging T1-T2a and PSA $<10 \mathrm{ng} / \mathrm{mL}$ and GS 6, Age range from $51-69$ and ECOG Performance Score for patients was 0 - 1.

\subsection{Acquisition and Simulation}

All patients underwent a computed tomography (CT) scan with $2 \mathrm{~mm}$ slice thickness. After the patients scanning the CT images has transferred to focal contouring system to delineate Target volume and organs at risk on the CT images on each axial slice then CT slices were transferred to treatment planning system.

\subsection{Planning Systems and Radiotherapy Machine}

Computerized Medical Systems (CMS) Inc.'s (St. Louis, MO) XiO software release 4.64 that incorporates modern dose calculation algorithms for dose calculation was used to plan cancer patients using Siemens Artiste linear accelerator, (was used in this work. It is a multienergy machine) 6 and 15 MV operating up to $900 \mathrm{MU} / \mathrm{min}$

Selected fifteen patients with early stage of prostate cancer to be planned with 3DCRT, ARC and IMRT. The tolerance level for OARs According to the Radiation Therapy Oncology Group (RTOG) protocol 0126. The beam arrangement was determined by the size and location of the tumor for 3DCRT, ARC plans and IMRT plans. Prescription dose and normal-tissue constraints were identical for the 3DCRT, ARC and IMRT plans. The total prescription dose to the PTV was 76 Gy delivered in 38 fractions at 2 Gy per fraction treating daily, five days per week.

\subsection{D-CRT, ARC and IMRT Technique}

For the three techniques we used different beam arrangement, energy and number of beams depending on the recommendation for each technique to give better coverage and spare for organs at risk. 3D-CRT, ARC and IMRT data are displayed in Table 1.

\subsection{Treatment Planning Evaluation}

The new treatment planning systems have many tools for qualitative and quantitative evaluation of the treatment plans. The visual slice-by slice review of the treatment plans using isodose lines distribution can be used as a qualitative evaluation for the treatment plans. The qualitative evaluation is important to know the location of the hot and cold areas in the treatment plans. The quantitative evaluation included the maximum, minimum, mean doses, the dose volume histograms (DVHs) and several indices. Dose Volume Histogram (DVH) was generated

Table 1. To describe 3D-CRT, ARC and IMRT Technique.

\begin{tabular}{cccc}
\hline Technique & 3D-CRT & Arc therapy & IMRT \\
\hline Energy & 6 and 15 MV & $15 \mathrm{MV}$ & $6 \mathrm{MV}$ \\
No of beams & 6 fields & 2 arcs & 7 fields \\
Beam arrangement & 4 oblique and 2 lateral & 2 oblique & spread around target with equal space \\
\hline
\end{tabular}


to evaluate the dose to the different structures in different treatment plans.

1) Dose Volume Histogram was generated to evaluate the mean dose to OARs and tolerance dose according to the Radiation Therapy Oncology Group (RTOG) protocol 0126 dose for rectum, bladder, left and right femoral head.

2) Cancer risk models for Radiation-Induced Cancer, The radiation risk model developed by ICRP Publication 103 for use in its recommendations. Excess relative risk (ERR) and Excess absolute risk (EAR) models were developed for cancer incidence and mortality incidence as a function of age at exposure.

$$
\begin{aligned}
& \mathrm{ERR}=\beta s D \cdot \exp [\gamma(e-30)+\eta \log (a / 70)] \\
& \mathrm{EAR}=\beta s D \cdot \exp [\gamma(e-30)-\eta \log (a / 70)]
\end{aligned}
$$

where $\beta s$ is referred to $\beta$ male or $\beta$ female which means sex specific estimation of ERR per Sv. $D=$ mean organ dose (Sv), $e=$ age at exposure (years) and $a=$ attained age (years), The coefficients $\beta$ male, $\beta$ female, $\gamma, \eta$, are given in tables 4.2 and 4.3 of ICRP Publication 103 in terms of cancer incidence [10].

It's a dosimetric study so there is no follow up taken to check difference between plans.

\section{Results and Discussion}

Estimation of second cancer risk calculation based on equivalent dose calculation from mean absorbed dose for fifteen patients with early stage prostate cancer with three different plans (3DCRT, Arc and IMRT) for rectum, bladder, left and right femoral head.

\subsection{Absorbed Dose (Gy)}

The mean dose in Gy and the SD for 3D-CRT for rectum, bladder, left and right femoral head for 3D-CRT were $(38.08 \pm 4.6),(44.33 \pm 5.45),(25.39 \pm 3.73)$ and $(26.72 \pm 4.01)$, respectively, For ARC (39.23 \pm 4.44$),(46.23 \pm 5.41),(34.39 \pm$ $3.78)$ and $(32.55 \pm 3.98)$, respectively and For IMRT, the doses were $((31.47 \pm$ $3.99),(38.01 \pm 5.12),(25.1 \pm 3.62)$ and $(23.64 \pm 4.5)$, respectively. Figure 1 shows average absorbed doses in rectum, bladder, right femoral and left femoral for 3D-CRT, ARC and IMRT technique.

The mean doses of the rectum, bladder were found to be significantly lower in IMRT plans Than 3DCRT plans and ARC plans with $\mathrm{P}<0.0000239$ for rectal mean dose, $\mathrm{P}<0.0003229$ for bladder mean dose. However, IMRT technique produced significantly lower mean dose for both the left and right femoral head than ARC with $\mathrm{P}<1.7602 \mathrm{E}-07$ and $\mathrm{P}<3.6482 \mathrm{E}-06$ for left and right femoral mean dose respectively but not significant in 3DCRT technique mean dose with $\mathrm{P}<0.8288$ mean dose for left femoral and $\mathrm{P}<0.06$ for right femoral mean dose.

\subsection{Excess Relative Risk (ERR)}

Figures 2-5 represent the Excess relative risk for rectum, bladder, left and right femoral head between 3D-CRT, ARC and IMRT as a function of age at exposure 


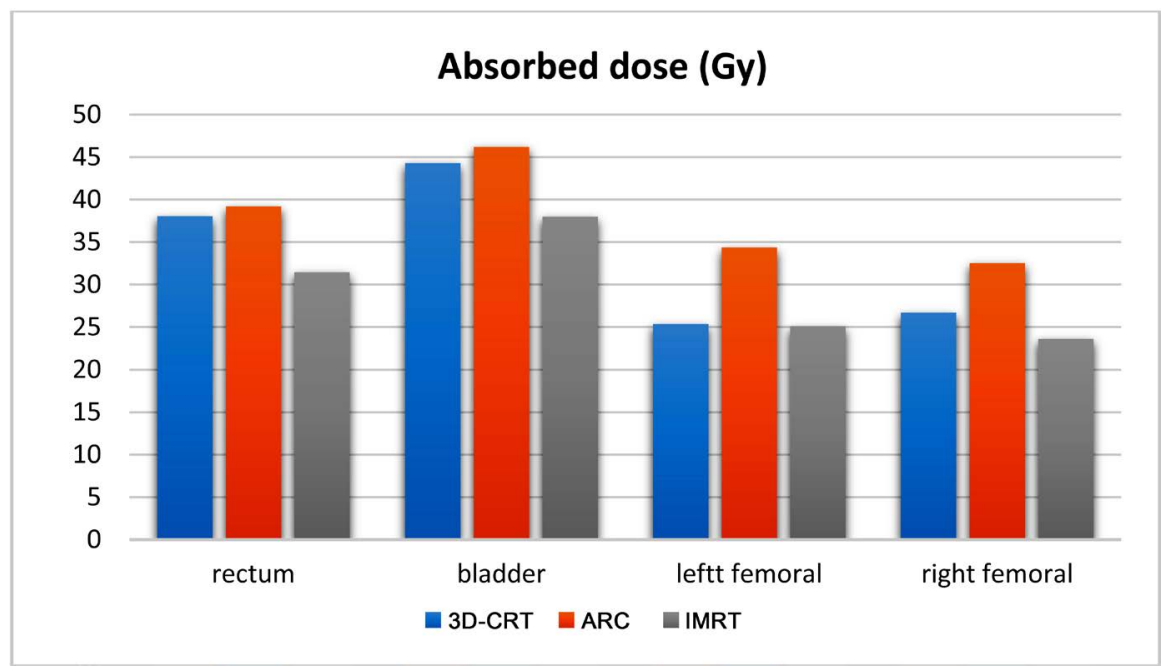

Figure 1. Average absorbed doses in rectum, bladder, right femoral and left femoral for 15 prostate patients treated using 3D-CRT, ARC and IMRT technique.

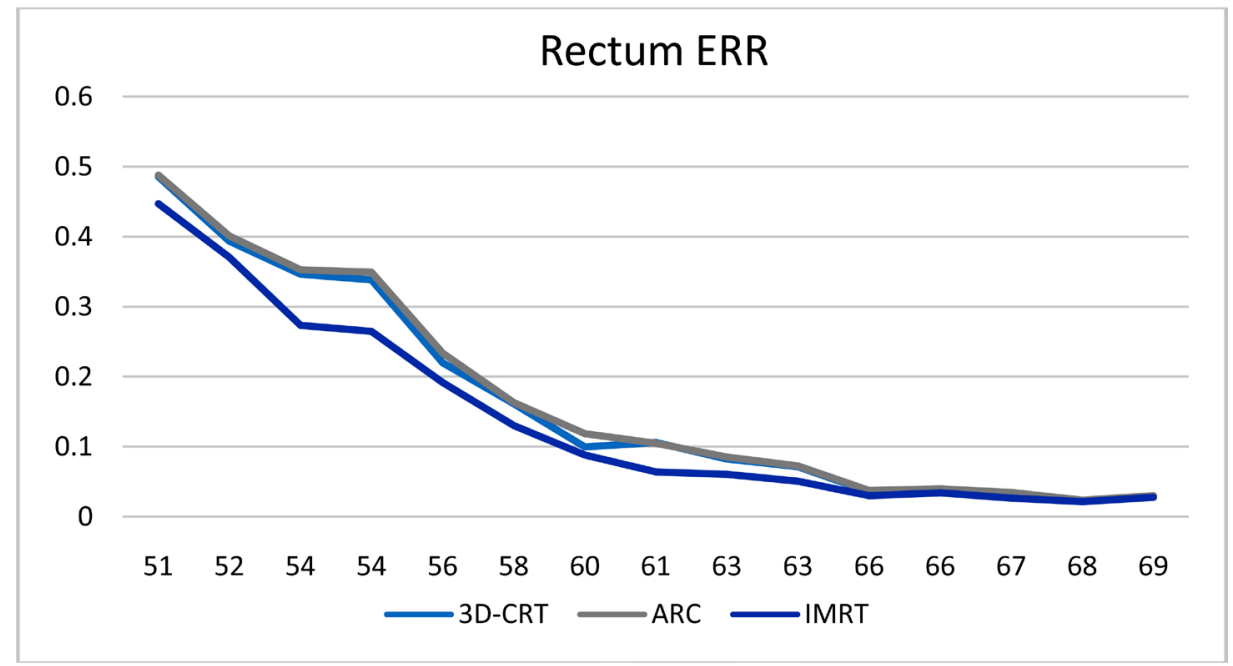

Figure 2. The excess relative risk with age of exposure for rectum in 3D-CRT, ARC and IMRT.

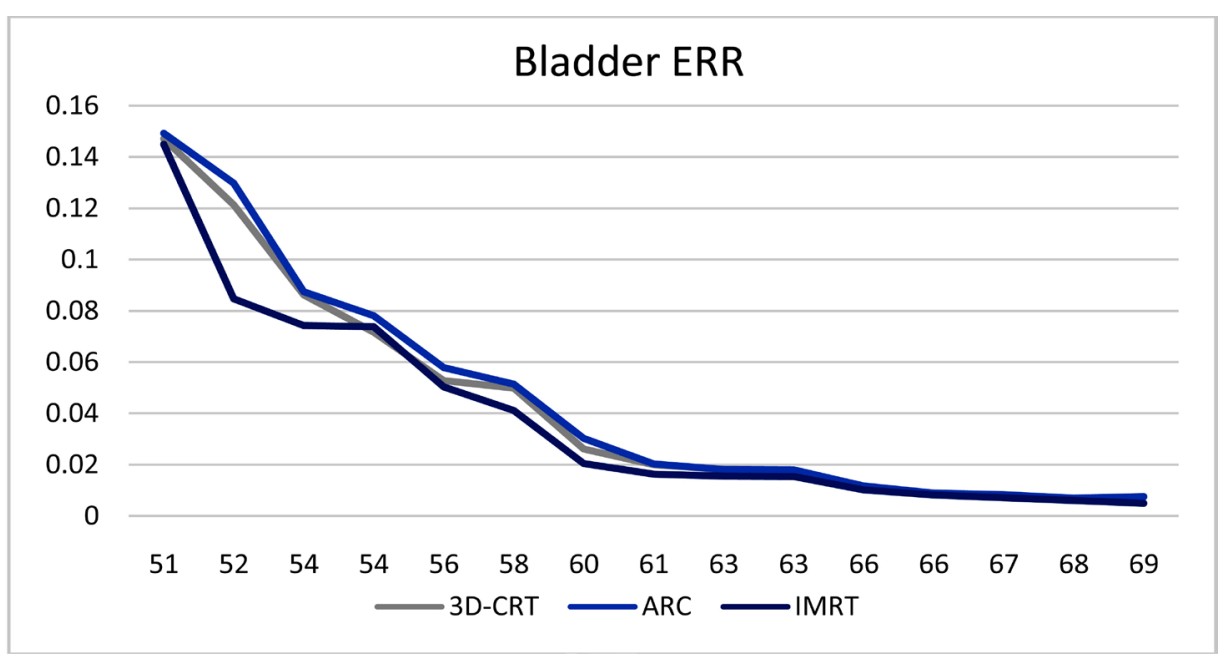

Figure 3. The excess relative risk with age of exposure for bladder in3D-CRT, ARC and IMRT. 


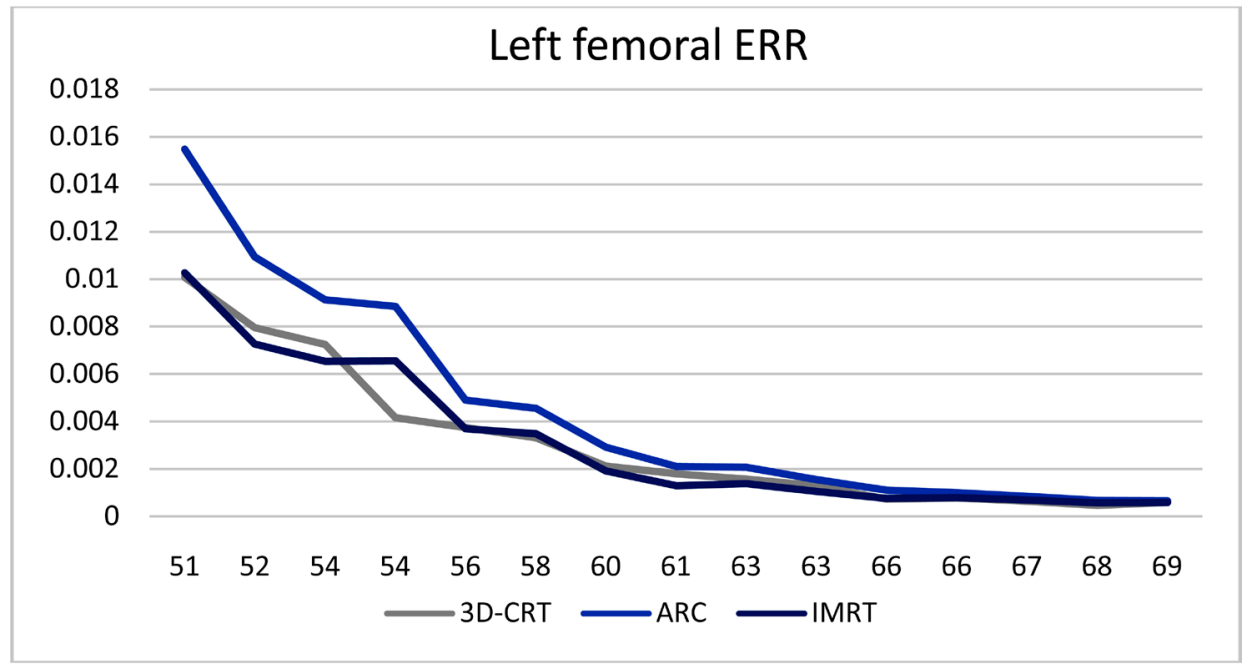

Figure 4. The excess relative risk with age of exposure for left femoral in 3D-CRT, ARC and IMRT.

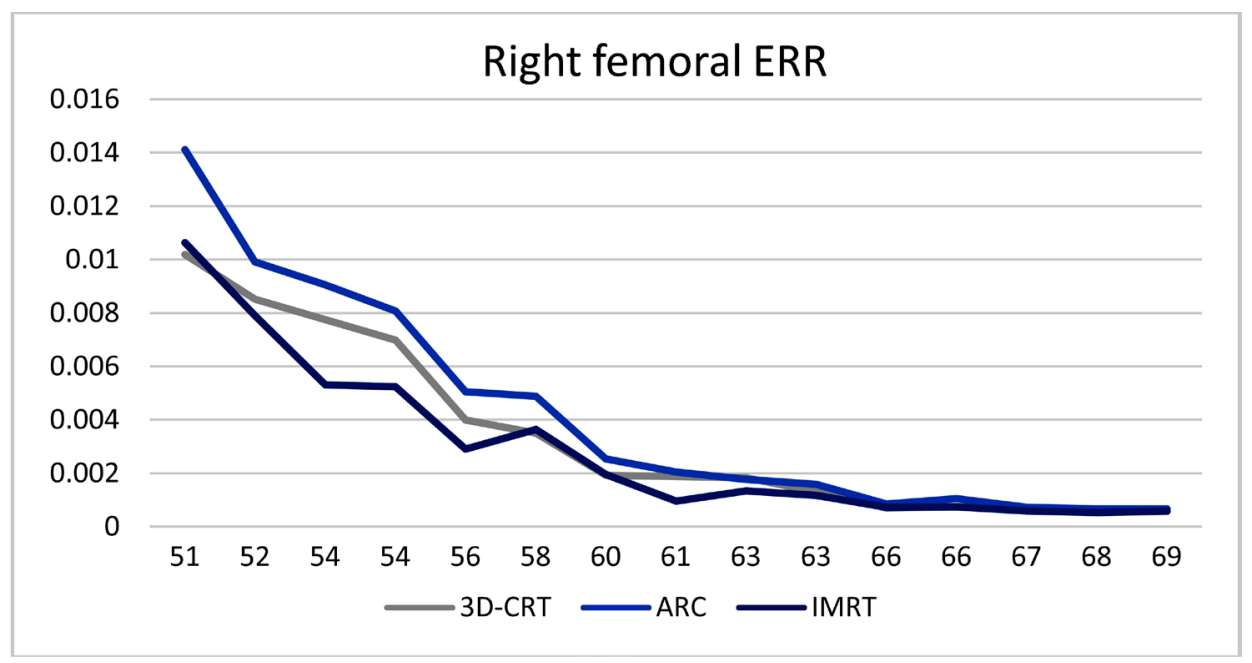

Figure 5. The excess relative risk with age of exposure for right femoral in 3D-CRT, ARC and IMRT.

Table 2. P-value for ERR model for second cancer risk in 3D-CRT, ARC and IMRT.

\begin{tabular}{ccccc}
\hline OAR/P-Value ERR & 3D-ARC & 3D-IMRT & ARC-IMRT & 3D-ARC-IMRT \\
\hline Rectum & 0.938422098 & 0.638841341 & 0.584693318 & 0.84180342 \\
Bladder & 0.902337661 & 0.732201958 & 0.643341806 & 0.891823405 \\
Left femoral & 0.349662023 & 0.987755437 & 0.430102543 & 0.513184744 \\
Right femoral & 0.567978178 & 0.703954285 & 0.361927763 & 0.627631072 \\
\hline
\end{tabular}

and by putting attained age 70 years old.

From Figures 2-5 we notice that Excess relative risk for OARs (rectum, bladder, left and right femoral head) decrease with increasing age of exposure and from Table 2 we conclude that there is no significant difference for ERR model for second cancer risk in 3D-CRT, ARC and IMRT. 


\subsection{Excess Absolute Risk (EAR)}

Figures 6-9 represent the Excess Absolute Risk (EAR) for rectum, bladder, left and right femoral head between 3D-CRT, ARC and IMRT as a function of age at exposure and by putting attained age 70 years old.

From Figures 6-9 we notice that Excess Absolute Risk for OARs (rectum, bladder, left and right femoral head) decrease with increasing age of exposure and from Table 3 we conclude that there is no significant difference for EAR model for second cancer risk in 3D-CRT, ARC and IMRT.

VMAT technique in published dosimetric studies has allowed coverage of target volumes, acceptable and comparable to that introduced by conventional IMRT using fixed or stationary fields [2] [11] [12].

In Palma et al. study Significant reduction of the dose to OAR by VMAT and IMRT compared to 3D conformal radiation therapy, where lowest doses with VMAT [2] [13].

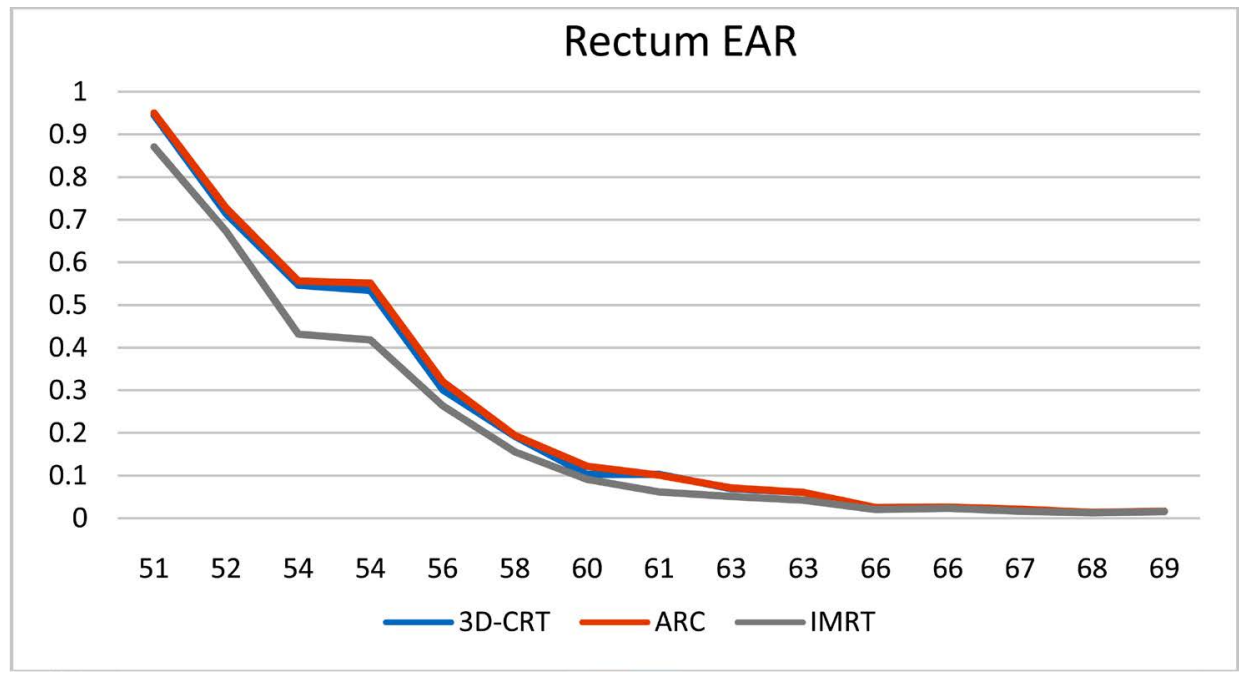

Figure 6. The excess absolute risk with age of exposure for rectum in 3D-CRT, ARC and IMRT.

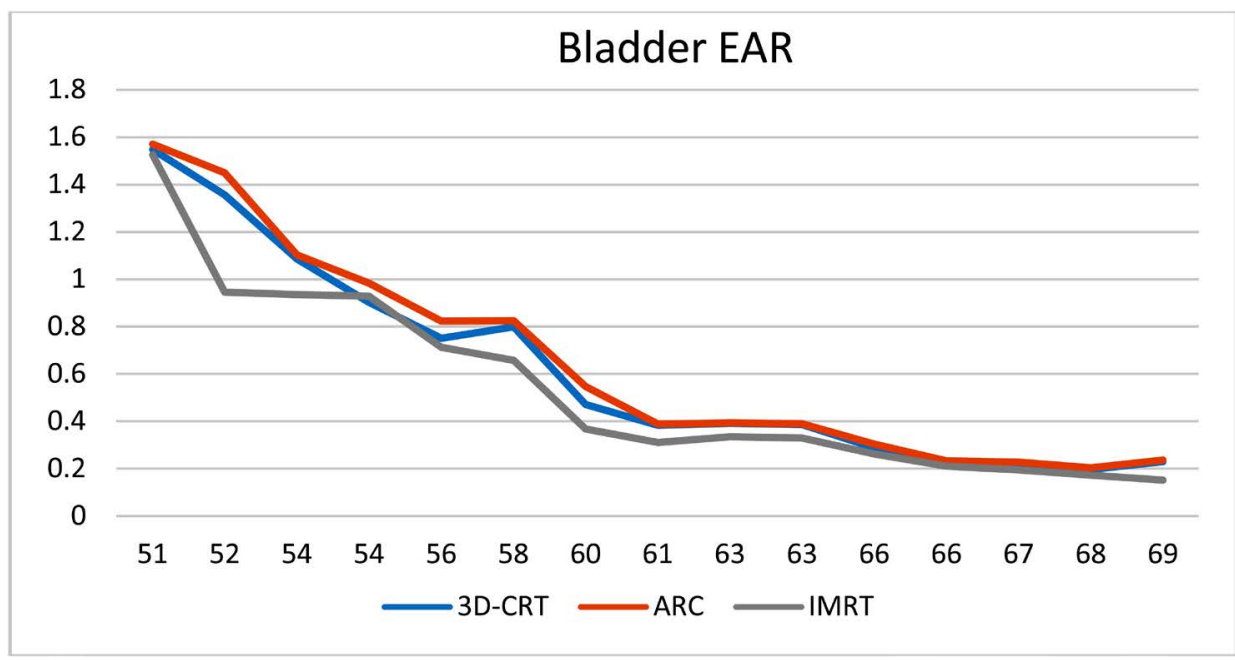

Figure 7. The excess absolute risk with age of exposure for bladder in 3D-CRT, ARC and IMRT. 


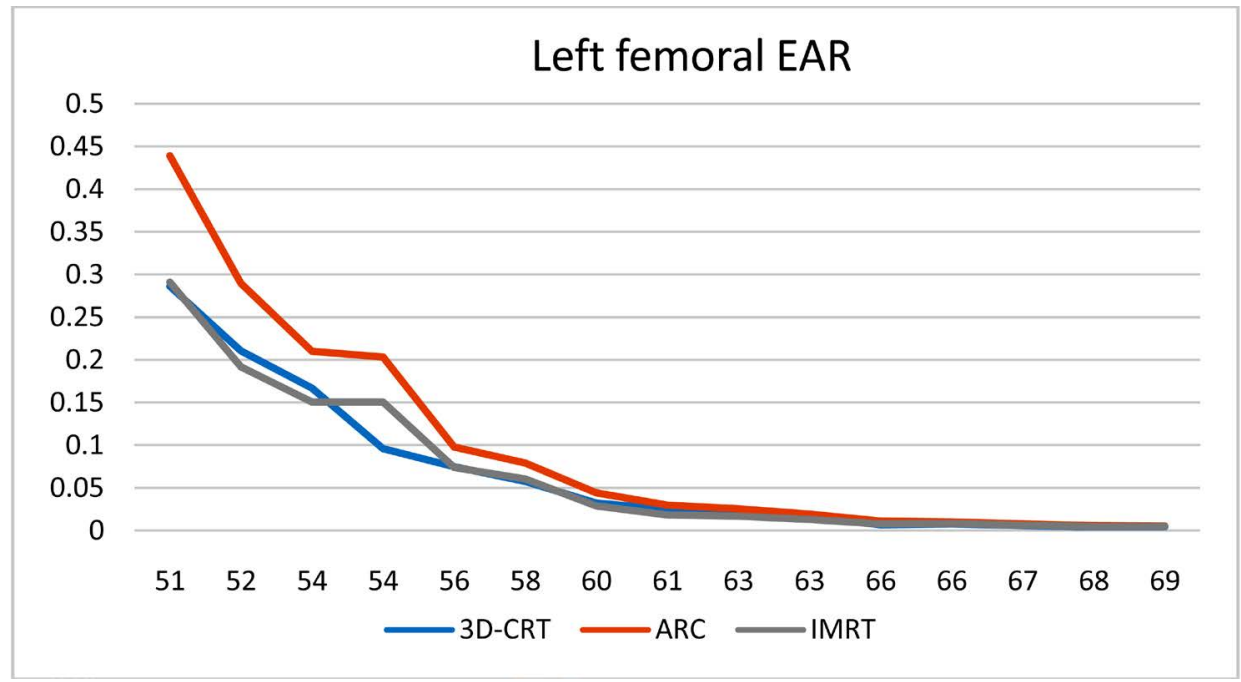

Figure 8. The excess absolute risk with age of exposure for left femoral in 3D-CRT, ARC and IMRT.

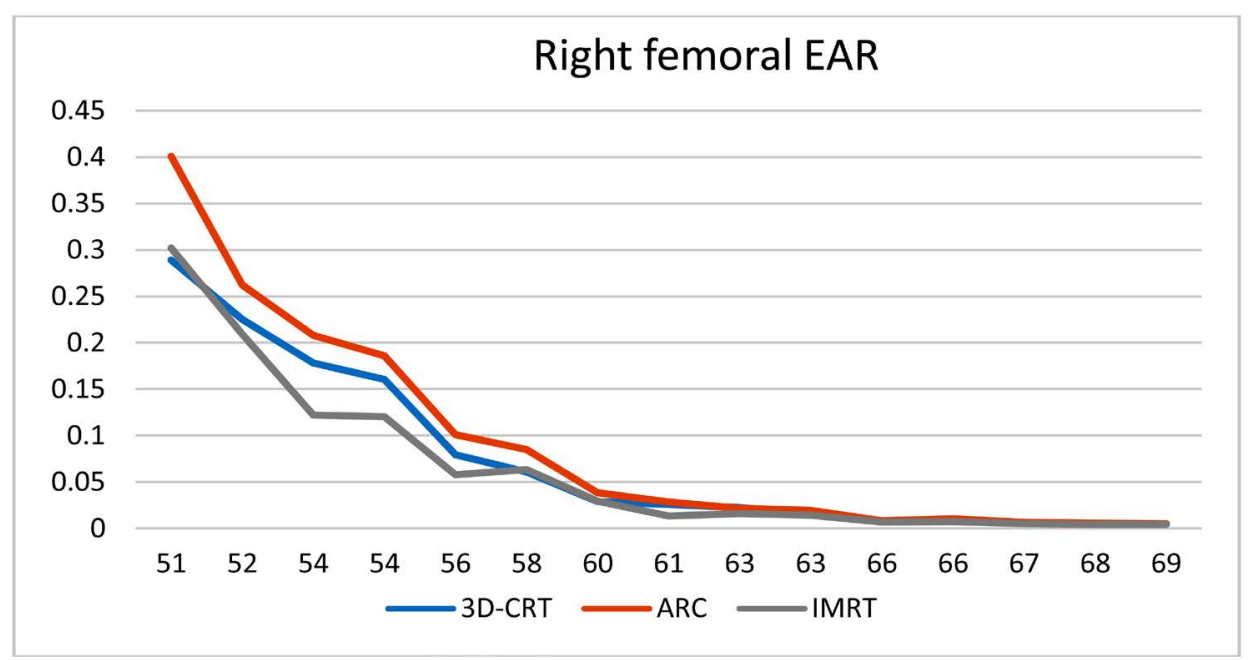

Figure 9. The excess absolute risk with age of exposure for right femoral in 3D-CRT, ARC and IMRT.

Table 3. P-value for EAR model for second cancer risk in 3D-CRT, ARC and IMRT.

\begin{tabular}{ccccc}
\hline OAR/P-Value EAR & 3D-ARC & 3D-IMRT & ARC-IMRT & 3D-ARC-IMRT \\
\hline Rectum & 0.700336181 & 0.740300577 & 0.700336181 & 0.91786803 \\
Bladder & 0.860662687 & 0.608703751 & 0.495337059 & 0.778172868 \\
Left femoral & 0.451481579 & 0.979601048 & 0.464576901 & 0.65188177 \\
Right femoral & 0.647153374 & 0.783297092 & 0.482737303 & 0.755544233 \\
\hline
\end{tabular}

Memorial Sloan Kettering Cancer Center comparative study reported a significant reduction of the dose to the rectum also the doses to the bladder and the femoral heads were also reduced but not significantly. Similar results by Hardcastle et al., that VMAT versus IMRT reduces doses to the rectum and therefore a lower rectum-NTCP [2] [14] [15] [16]. 
Murray et al. [17] investigated that SPC risk from modern EBRT by comparing: 3D-CRT, IMRT, volumetric arc therapy (VMAT), and stereotactic ablative radiotherapy (SABR), the excess absolute risks have been determined for out-of-field organs, organ equivalent dose calculations and a linear model was employed for the estimation of the excess absolute risk. 3D-CRT Compared to both IMRT and VMAT showed an increased risk in several out-of-field organs, by up to $26 \%$ and $55 \%$, respectively. The absolute risks were low for all irradiation methods, and so were the absolute differences among technique [18].

\section{Conclusions}

The results showed mean doses of the rectum, bladder with IMRT lower than 3DCRT plans and ARC therapy plans. But for femoral heads mean dose with IMRT is lower than rotational but not in 3DCRT technique.

The second cancer risk models (ERR and EAR) for OARs decrease with increasing age of exposure for 3D-CRT, ARC and IMRT and there is no significant difference for ERR and EAR model for developing second cancer risk in 3D-CRT, ARC and IMRT.

\section{Acknowledgements}

This work was supported by Ayady Al Mostakbal Oncology Center, Alexandria, Egypt. We would like to thank Dr. Assem Rostom, MD, FRCR General Manager and Consultant Clinical Oncologist Ayadi Al-Mostakbal Charity Clinical Oncology Center for encourage and supporting all the time.

\section{References}

[1] Bakiu, E., Telhaj, E., Kozma, E., Ruçi, F. and Malka, J.P. (2013) Comparison of 3D CRT and IMRT Tratment Plans. Acta Informatica Medica, 21, 211-212. https://doi.org/10.5455/aim.2013.21.211-212

[2] Lalya, I., Zaghba, N., Andaloussi-Saghir, K., Elmarjany, M., Baddouh, L., Dahmani, K., Hadadi, K., Sifat, H. and Mansouri, H. (2016) Volumetric Modulated Arc Therapy versus Intensity Modulated Radiation Therapy in the Treatment of Prostate Cancer: A Systematic Literature Review. International Journal of Radiation Oncology Biology Physics, 2, 15-20. https://doi.org/10.17352/ijrro.000014

[3] Wallis, C., Mahar, A., Choo, R., Herschorn, S., Kodama, R., Shah, P., Danjoux, C., Narod, S. and Nam, R. (2016) Second Malignancies after Radiotherapy for Prostate Cancer: Systematic Review and Meta-Analysis. BMJ, 352, 851. https://doi.org/10.1136/bmj.i851

[4] Cakir, A., Akgun, Z., Fayda, M. and Agaoglu, F. (2015) Comparison of Three Dimensional Conformal Radiation Therapy, Intensity Modulated Radiation Therapy and Volumetric Modulated Arc Therapy for Low Radiation Exposure of Normal Tissue in Patients with Prostate Cancer. Asian Pacific Journal of Cancer Prevention, 16, 3365-3370. https://doi.org/10.7314/APJCP.2015.16.8.3365

[5] Stankovic, V., Zamic, Z., Pekmezovic, T., Tepavcevic, D.K., Dozic, M., Saric, M., Vuckovic, S. and Nikitovic, M. (2016) Acute and Late Genitourinary Toxicity after 72 Gy of Conventionally Fractionated Conformal Radiotherapy for Localised Prostate Cancer: Impact of Individual and Clinical Parameters. Journal of Clinical On- 
cology, 28, 577-586. https://doi.org/10.1016/j.clon.2016.04.041

[6] Halperin, E.C., Perez, C.A. and Brady, L.W. (2008) Perez and Brady's Principles and Practice of Radiation Oncology. Lippincott Williams \& Wilkins, Philadelphia.

[7] Jolnerovski, M., Salleron, J., Beckendorf, V., Peiffert, D., Baumann, A.-S., Bernier, V., Huger, S., Marchesi, V. and Chira, C. (2017) Intensity-Modulated Radiation Therapy from 70 Gy to 80 Gy in Prostate Cancer: Six-Year Outcomes and Predictors of Late Toxicity. Radiation Oncology, 12, 99. https://doi.org/10.1186/s13014-017-0839-3

[8] Contesini, M., Guberti, M., Saccani, R., Braglia, L., Iotti, C., Botti, A., Abbati, E. and Iemmi, M. (2017) Setup Errors in Patients with head-neck cancer (HNC), Treated Using the Intensity Modulated Radiation Therapy (IMRT) Technique: How It Influences the Customised Immobilisation Systems, Patient's Pain and Anxiety. Radiation Oncology, 12, 72. https://doi.org/10.1186/s13014-017-0807-y

[9] Tran, A., Zhang, J., Woods, K., Yu, V., Nguyen, D., Gustafson, G., Rosen, L. and Sheng, K. (2017) Treatment Planning Comparison of IMPT, VMAT and $4 \pi$ Radiotherapy for Prostate Cases. Radiation Oncology, 12, 10. https://doi.org/10.1186/s13014-016-0761-0

[10] International Commission on Radiological Protection, (2012) ICRP Publication 103: Low-Dose Extrapolation of Radiation-Related Cancer Risk. Annals of the ICRP, 37, 1-328.

[11] Peeters, S.T., Heemsbergen, W.D., Koper, P.C., van Putten, W.L., Slot, A., et al. (2006) Dose-Response in Radiotherapy for Localized Prostate Cancer: Results of the Dutch Multicenter Randomized Phase III Trial Comparing 68 Gy of Radiotherapy with 78 Gy. Journal of Clinical Oncology, 24, 1990-1996. https://doi.org/10.1200/JCO.2005.05.2530

[12] Beckendorf, V., Guérif, S., Le Prisé, E., Cosset, J.M., Lefloch, O., et al. (2004) The GETUG 70 Gy vs. 80 Gy Randomized Trial for Localized Prostate Cancer: Feasibility and Acute Toxicity. International Journal of Radiation Oncology Biology Physics, 60, 1056-1065. https://doi.org/10.1016/j.ijrobp.2004.05.033

[13] Beckendorf, V., Guerif, S., Le Prise, E., Cosset, J., Bougnoux, A., et al. (2008) 70 Gy versus (vs) 80 Gy Dose Escalation Getug 06 French Trial for Localized Prostate Cancer: Mature Results. International Journal of Radiation Oncology Biology Physics, 72, S96-S97. https://doi.org/10.1016/j.ijrobp.2008.06.986

[14] Ling, C.C., Burman, C., Chui, C.S., Kutcher, G.J., Leibel, S.A., et al. (1996) Conformal Radiation Treatment of Prostate Cancer Using Inversely-Planned Intensity-Modulated Photon Beams Produced with Dynamic Multileaf Collimation. International Journal of Radiation Oncology Biology Physics, 35, 721-730.

https://doi.org/10.1016/0360-3016(96)00174-5

[15] Damen, E.M., Brugmans, M.J., van der Horst, A., Bos, L., Lebesque, J.V., et al. (2001) Planning, Computer Optimization, and Dosimetric Verification of a Segmented Irradiation Technique for Prostate Cancer. International Journal of Radiation Oncology Biology Physics, 49, 1183-1195. https://doi.org/10.1016/S0360-3016(00)01525-X

[16] De Meerleer, G.O., Vakaet, L.A., De Gersem, W.R., De Wagter, C., De Naeyer, B., et al. (2000) Radiotherapy of Prostate Cancer with or without Intensity Modulated Beams: A Planning Comparison. International Journal of Radiation Oncology Biology Physics, 47, 639-648. https://doi.org/10.1016/S0360-3016(00)00419-3

[17] Murray, L.J., Thompson, C.M., Lilley, J., Cosgrove, V., Franks, K., Sebag-Montefiore, D., et al. (2015) Radiation-Induced Second Primary Cancer Risks from Modern Ex- 
ternal Beam Radiotherapy for Early Prostate Cancer: Impact of Stereotactic Ablative Radiotherapy (SABR), Volumetric Modulated Arc Therapy (VMAT) and Flattening Filter Free (FFF) Radiotherapy. Physics in Medicine \& Biology, 60, 1237-1257. https://doi.org/10.1088/0031-9155/60/3/1237

[18] Bezak, E., Takam, R., Yeoh, E., Loredana, G.M. (2017) The Risk of Second Primary Cancers Due to Peripheral Photon and Neutron Doses Received during Prostate Cancer External Beam Radiation Therapy. Physica Medica, 42, 253-258.

https://doi.org/10.1016/j.ejmp.2017.02.018 